\title{
Stem Cells, Cell Therapies, and Bioengineering in Lung Biology and Disease 2019
}

\author{
Darcy E. Wagner (1) ${ }^{1,15}$, Laertis Ikonomou ${ }^{2,15}$, Sarah E. Gilpin (10 ${ }^{3}$, \\ Chelsea M. Magin ${ }^{4}$, Fernanda Cruz ${ }^{5}$, Allison Greaney ${ }^{6}$, Mattias Magnusson ${ }^{7}$, \\ Ya-Wen Chen ${ }^{8}$, Brian Davis ${ }^{9}$, Kim Vanuytsel $^{2}$, Sara Rolandsson Enes ${ }^{10,11}$, \\ Anna Krasnodembskaya (10 ${ }^{12}$, Mareike Lehmann (10 ${ }^{13}$, \\ Gunilla Westergren-Thorsson ${ }^{11}$, John Stegmayr ${ }^{1}$, Hani N. Alsafadi ${ }^{1}$, \\ Evan T. Hoffman ${ }^{10}$, Daniel J. Weiss ${ }^{10}$ and Amy L. Ryan (10) 8,14
}

ABSTRACT A workshop entitled "Stem Cells, Cell Therapies and Bioengineering in Lung Biology and Diseases" was hosted by the University of Vermont Larner College of Medicine in collaboration with the National Heart, Lung and Blood Institute, the Alpha-1 Foundation, the Cystic Fibrosis Foundation, the International Society for Cell and Gene Therapy and the Pulmonary Fibrosis Foundation. The event was held from July 15 to 18, 2019 at the University of Vermont, Burlington, Vermont. The objectives of the conference were to review and discuss the current status of the following active areas of research: 1) technological advancements in the analysis and visualisation of lung stem and progenitor cells; 2) evaluation of lung stem and progenitor cells in the context of their interactions with the niche; 3 ) progress toward the application and delivery of stem and progenitor cells for the treatment of lung diseases such as cystic fibrosis; 4) progress in induced pluripotent stem cell models and application for disease modelling; and 5) the emerging roles of cell therapy and extracellular vesicles in immunomodulation of the lung. This selection of topics represents some of the most dynamic research areas in which incredible progress continues to be made. The workshop also included active discussion on the regulation and commercialisation of regenerative medicine products and concluded with an open discussion to set priorities and recommendations for future research directions in basic and translation lung biology.

@ERSpublications

This workshop report discusses recent advances in cell therapy and bioengineering approaches for repair and regeneration of diseased lungs https://bit.ly/2DqA8eu

Cite this article as: Wagner DE, Ikonomou L, Gilpin SE, et al. Stem Cells, Cell Therapies, and Bioengineering in Lung Biology and Disease 2019. ERJ Open Res 2020; 6: 00123-2020 [https:// doi.org/10.1183/23120541.00123-2020].
This article has supplementary material available from openres.ersjournals.com.

Received: 9 March 2020 | Accepted after revision: 31 July 2020

Copyright $\odot$ ERS 2020. This article is open access and distributed under the terms of the Creative Commons Attribution Non-Commercial Licence 4.0. 


\section{Introduction}

Following the inaugural conference, held in 2005 and founded by D.J. Weiss (University of Vermont) and D. Prockop (Texas A\&M University), the eighth biennial conference, held at the University of Vermont, provided the most current discussion of recent advances in the field of lung stem cells, cellular therapies and bioengineering (figure 1). For the second successive conference D.E. Wagner (Lund University, Sweden) chaired, with A.L. Ryan (Firth) (University of Southern California) and L. Ikonomou (Boston University) serving as co-vice chairs.

Application of new and emerging technologies to lung stem cell biology and regeneration has led to exciting advances in the field. Two central themes that emerged at the 2019 conference were: 1) innovation and application of new technology for advancing basic and translational lung biology and 2) the impact of niche/microenvironment on cellular regulation. The necessity for basic science studies to enhance fundamental understanding of lung regeneration and to design innovative translational studies to move the field closer to regenerative strategies for acute and chronic lung diseases was also reiterated.

As a cogent example of evolving technologies, single-cell RNA sequencing (scRNA-seq) is rapidly becoming a technique used in research laboratories all over the world. While the value of scRNA-seq in hypothesis formation cannot be understated, further advances in informatics techniques for utilisation of the data is essential to gain a greater understanding of potential functional implications. The capacity for data integration across multiple species and collaborative real-time data exploration in multiple laboratories was highlighted with the use of three-dimensional (3D) visualisation of datasets, enabling physical interaction with the data without the constraints of two-dimensional (2D) space [1]. New computational approaches to data mining have also enabled systems-level approaches for discovering putative ligand-receptor interactions and the identification of cell signalling pathways and networks [2]. These new techniques hold great promise for improving our understanding of lung regenerative biology and its potential for clinical translation.

Recent developments in ex vivo cell expansion techniques, improved physiological 3D models and culturing techniques, greater understanding of endogenous lung stem and progenitor cell behaviour and improved pluripotent stem cell differentiation protocols all increase the hope for success in the pursuit of functional long-term cell engraftment into the lung and the generation of functional tissue for transplantation [3,4]. New technologies such as scRNA-seq have helped elucidate the substantial cellular heterogeneity within the human lung. scRNA-seq also opens new doors for further understanding how similar ex vivo cultured primary cells, such as airway basal epithelial cells and induced pluripotent stem cell (iPSC)-derived lung cells are to the resident endogenous cells of the adult human lung. This remains an area of continued active discussion and investigation. Furthermore, differences in protocols across laboratories continue to make direct comparisons challenging.

For cell engraftment to be plausible in the human lung it is essential to have suitable models for pre-clinical evaluation prior to translation into humans. The use of the ferret as a model for cystic fibrosis (CF) was discussed at this conference in the context of the lung basal stem cells and their niche post-transplantation. Essential pre-clinical investigation into how to deliver cells and how to access the appropriate endogenous niche or how to mobilise endogenous cells are all being pursued in hope for repairing the airway epithelium to a functional status. Furthermore, understanding the complexity of the regulation of lung stem and progenitor cells by their direct, in vivo, niche is vital to the success of any

Affiliations: 'Lung Bioengineering and Regeneration, Dept of Experimental Medicine, Wallenberg Center for Molecular Medicine and Lund Stem Cell Center, Faculty of Medicine, Lund University, Lund, Sweden. ${ }^{2}$ Center for Regenerative Medicine, Boston University and Boston Medical Center, Boston, MA, USA. ${ }^{3}$ Wyss Institute for Biologically Inspired Engineering at Harvard University, Boston, MA, USA. ${ }^{4}$ Depts of Medicine and Bioengineering, University of Colorado, Denver, Aurora, CO, USA. ${ }^{5}$ Federal University of Rio de Janeiro, Rio de Janeiro, Brazil. ${ }^{6}$ Dept of Biomedical Engineering, Yale University, New Haven, CT, USA. ${ }^{7}$ Molecular Medicine and Gene Therapy, Lund Stem Cell Center, Lund University, Lund, Sweden. ${ }^{8}$ Hastings Center for Pulmonary Research, Dept of Medicine, University of Southern California, Los Angeles, CA, USA. ${ }^{9}$ Center for Stem Cell and Regenerative Medicine, Brown Foundation Institute of Molecular Medicine, University of Texas Health Science Center, Houston, TX, USA. ${ }^{10}$ Dept of Medicine, University of Vermont, Burlington, VT, USA. ${ }^{11}$ Dept of Experimental Medical Science, Division of Lung Biology, Lund University, Lund, Sweden. ${ }^{12}$ Wellcome-Wolfson Research Institute, Queen's University Belfast, Belfast, UK. ${ }^{13}$ Comprehensive Pneumology Center, Lung Repair and Regeneration Unit, Helmholtz Center Munich, Munich, Germany. ${ }^{14}$ Dept of Stem Cell and Regenerative Medicine, University of Southern California, Los Angeles, CA, USA. ${ }^{15}$ These authors contributed equally.

Correspondence: Amy L. Ryan (Firth), Hastings Center for Pulmonary Research, Division of Pulmonary, Critical Care and Sleep Medicine, Dept of Medicine, Dept of Stem Cell Biology and Regenerative Medicine, HMR 712, University of Southern California, Los Angeles, CA 90089, USA. E-mail: amy.firthamed.usc.edu 

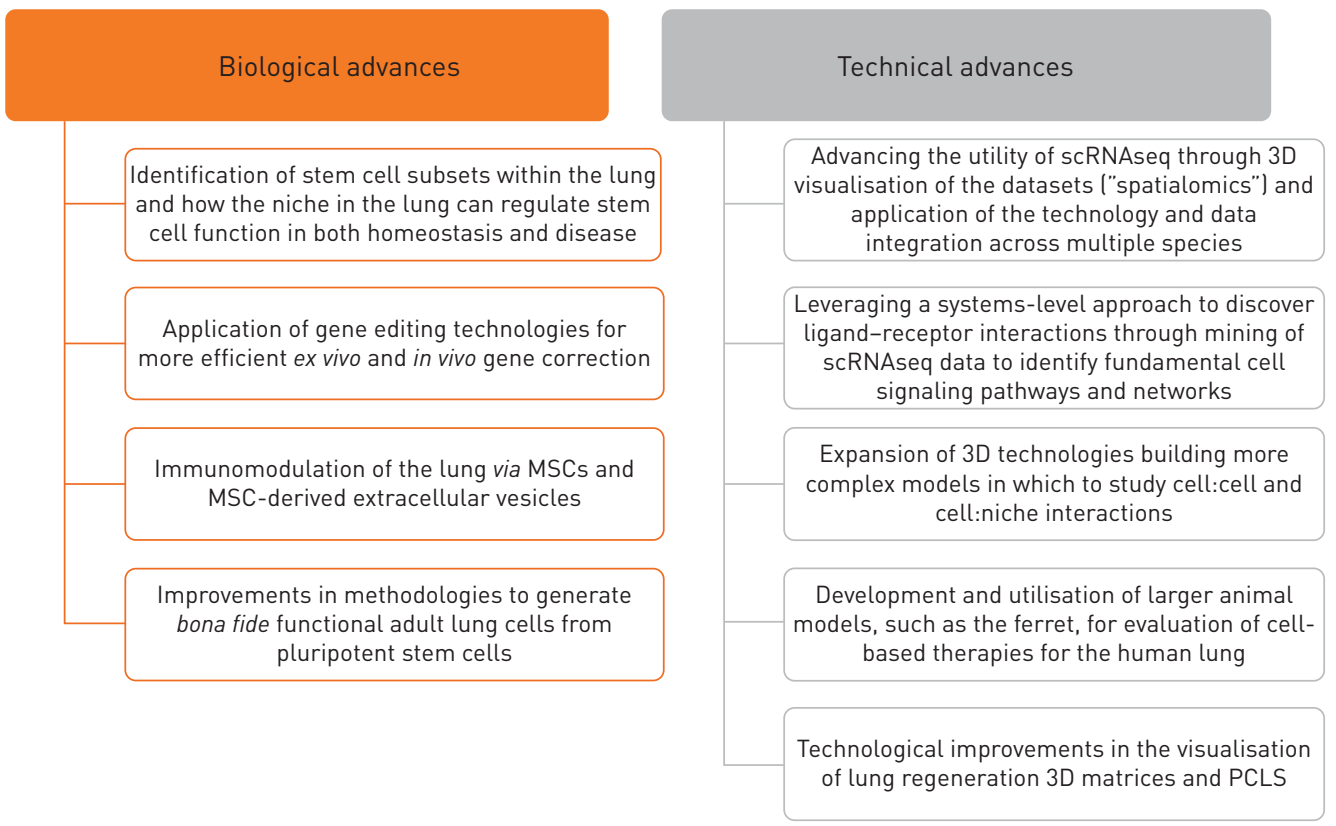

FIGURE 1 Scientific advances and application of innovative and new technologies and techniques in human lung regeneration. The Stem Cells, Cell Therapies and Bioengineering in Lung Biology and Diseases 2019 conference was the eighth in a series of biennial conferences focusing on advances in biotechnology and bioengineering, endogenous lung stem/progenitor cells and cell-based therapies, increasing our knowledge of lung stem cell populations, and edging closer to addressing the barriers toward making cell therapy feasible in the epithelial and vascular compartments of the lung. Significant advances since the last conference in 2017, are summarised in table 1. MSC: mesenchymal stromal cell; scRNA-seq: single-cell ribonucleic acid sequencing; 3D: three-dimensional; PCLS: precision-cut lung slice.

mechanism for restoration of homeostasis in a diseased lung. To this end, greater understanding of the impact of cell-cell, cell-niche and cell-matrix interactions within the normal and diseased lung is needed.

While the cell therapy field still faces many challenges, the clinical translation of mesenchymal stromal cell (MSC)-based therapies is an active area for respiratory focused translational studies. The field has been held back largely due to a scarcity in reliable and rapid disease-specific potency assays allowing statistically relevant prediction of therapeutic efficacy [5-8]. An emerging focus is on the immunomodulatory phenotypes that MSC-derived exosomes may exert in the diseased lung. Further functional elucidation of the role of extracellular vesicles, such as exosomes, may be instrumental in identification of novel therapeutic targets and mechanisms. Continued progress in this area will probably be dependent upon fully understanding the impact of disease-dependent changes in the lung microenvironment. Despite progress in understanding the biology underpinning the efficacy of cell therapies in pre-clinical models, including understanding of how the microenvironment influences effective cell therapy [9], there is currently no clinically approved cellular therapy for lung disease. However, there remains an alarming rate of patients receiving unproven and unregulated cellular interventions, which raises a number of clinical, ethical and regulatory concerns [10-12].

Importantly, the 2019 conference continued discussion to develop an actionable agenda for the prohibition of unproven and unlicensed stem cell therapies that was initiated at the 2017 conference [13]. All of the above-mentioned topics were featured in selected symposia at the conference and presented as active topics for discussion. The conference again was paired with a "hands-on" pre-conference workshop focusing on training in state-of-the-art methods in stem cells, cell therapies and bioengineering. A range of existing and emerging techniques relevant to the field of lung bioengineering and regeneration were featured such as: decellularisation of whole lungs, preparation of precision cut lung slices (PCLSs), ex vivo culture techniques, four-dimensional imaging techniques, gene editing and virtual reality techniques for visualising scRNA-seq data. An additional new pre-conference workshop was introduced this year focusing on professional skill development in technology, commercialisation and business communication for academic researchers. The 2019 conference concluded with a series of survey questions addressing key theme areas of the conference. This report summarises the topics featured throughout the conference, highlighting key areas for continued innovation and funding to enable the field to successfully advance. The full pre-conference and conference programme, as well as submitted abstracts, is included as a supplement. 


\section{Session I. Understanding lung stem cells in the digital age}

The development of scRNA-seq [14] has revolutionised our capacity to evaluate cellular heterogeneity in complex tissues. This capability has led to the discovery of a rare cell population of ionocytes in the lung $[15,16]$. By evaluating lungs from multiple species with scRNA-seq, RAREDON et al. [2] (Yale University, USA) leveraged a systems-level approach to identify highly conserved cell signalling pathways. Potential ligand-receptor interactions were mined to identify these pathways, which are predicted to regulate adult lung homeostasis. Such approaches can now be applied to develop a translational roadmap identifying key regulatory mechanisms in homeostasis and disease, as well as for tissue engineering. scRNA-seq has further revolutionised our capabilities to study cellular mechanisms of lung development [17-20] and disease $[18,19,21,22]$

scRNA-seq data is usually visualised in 2D space using t-distributed stochastic neighbour embedding (t-SNE) or uniform manifold approximation and projection. CellexalVR, developed by S. Soneji (Lund University, Sweden), has expanded scRNA-seq data visualisation to $3 \mathrm{D}$ by developing an open-source virtual reality (VR) environment based on video-game technology [1]. The 3D cell maps can be moved and rotated like physical objects and hand-selected groups of cells can be compared directly in the same VR environment. CellexalVR represents a new way of working with large datasets without space constraints and can be utilised anywhere by multiple users simultaneously, thereby enriching collaborative data exploration.

scRNA-seq is also being applied to study regenerative processes. LEIGH and co-workers [23] (Harvard University, USA) leveraged this technology to study the dynamic cellular landscape during axolotl (Salamander) limb regeneration and homeostasis. scRNA-seq on various stages of this process were used to create computational predictions for the progression of limb regeneration over time. Cellular dynamics can also be computationally evaluated using scRNA-seq over pseudo-time. Pseudo-time analysis mechanisms were first introduced in 2014 [24, 25], followed by many other such programs [26]. In the Velocity method, data on spliced and unspliced mRNA sequences are leveraged to extrapolate dynamic lineage relationships among cells and predict progenitor cell fate [27].

Two major challenges remain with current scRNA-seq analyses. First, scRNA-seq data usually lack spatial orientation within the analysed tissue, as single cells are sequenced from a dissociated "soup". This can be resolved using new "spatialomics" techniques, such as multiplexed error-robust fluorescence in situ hybridisation, laser capture microscopy coupled with Smart-seq and deterministic barcoding in tissue for spatial omics sequencing [28-33]. Second, functional assays are necessary for verifying gene expression. This often involves histological staining or the use of fluorescent reporters [34]. Nonetheless, scRNA-seq particularly benefits the lung community in resolving significant cellular heterogeneity and plasticity in homeostasis and disease.

\section{Session II. Building up the complexity of the lung: assessing lung stem and progenitor cells using 3D models}

Efforts to recreate 3D tissues have been reinvigorated by 3D printing technology in the biomedical field, using biomaterial inks and bioinks, combining various biocompatible materials and cells, to create tissue models of hearts, blood vessels and tracheas. These printed constructs recapitulate key structural, mechanical and biological properties of native tissues. A functional tracheal graft, for example, must be biocompatible, bioactive and able to maintain proper structure and mechanical strength over time. FeINBERG and co-workers [35] (Carnegie Mellon University, USA) developed a 3D bioprinting technique, known as Freeform Reversible Embedding of Suspended Hydrogels (FRESH), in 2015 and presented an update to this technology. The FRESH system uses a bioink of collagen, or other materials, embedded in a thick slurry of gelatine microparticles and water. The new system is now able to print collagen with greater resolution and fidelity. This technique has been successfully applied to bio-print human heart structures at scales ranging from capillaries to a model of the entire organ [36] and FeINBERG's group is working to apply FRESH 2.0 methodology to bioprinted tracheas for paediatric patients. A potential pitfall to build up a human lung from, either using 3D printing techniques or decellularised lung scaffolds, is to generate sufficiently large quantities of progenitors or the appropriate mature cell type to achieve this endeavour.

2D culture and animal models have long been used to study human diseases; however, these conventional models have several limitations. Traditional 2D cultures lack proper spatial cues and cell complexity. Animal models, especially mouse models have been extremely useful in advancing our understanding of lung repair and regeneration. A Wnt-responsive alveolar epithelial progenitor lineage within the alveolar type 2 (AT2) cell population has been shown to act as a major facultative progenitor cell in mouse distal lung and human alveolar organoids [4]. However, given the significant difference between the human and mouse lung, especially in the distal airway, mouse models rarely completely recapitulating all aspects of the pathogenesis of human disease. As an alternative strategy, organoid and 3D cultures derived from human 
cells and tissue have been used more frequently. Gотон and co-workers [37, 38] (Kyoto University, Japan) used carboxypeptidase $\mathrm{M}(\mathrm{CPM})$ to isolate ventralised anterior foregut endoderm cells, derived from human iPSCs, with lung competence. $\mathrm{CPM}^{+}$cells can generate alveolar epithelial cells as well as airway epithelium in 3D cultures. Using this system, they modelled Hermansky-Pudlak Syndrome (HPS) type 2, an autosomal recessive disorder with dysfunctional lysosome-related organelles in AT2 cells, in alveolar spheroids derived from human iPSC generated from a patient with HPS type 2. This recapitulated key human disease features, including altered distribution, size and secretion pattern of the lamellar bodies, highlighting the potential of use of human pluripotent stem cell-derived alveolar spheroid models for studying alveolar diseases [39]. Gотон and co-workers also presented the generation of abnormal multi-ciliated airway spheroid structures using iPSCs derived from primary ciliary dyskinesia (PCD) patients, demonstrating that this model may provide a new diagnostic and research platform for difficult cases of PCD. Gотон and co-worker's research complements that of other groups $[40,41]$ who have been able to sort CD $47^{\text {high }} / \mathrm{CD} 26^{\text {low }}$ cells to purify NKX2.1 $1^{+}$lung epithelial progenitor cells capable of directed differentiation into alveolar spheres. iPSC-derived alveolar spheres have been used to model genetic alveolar disease using CRISPR/Cas9 [40]. Using reporter cell lines and scRNA-seq to map fate trajectories, they have demonstrated lung epithelial lineage heterogeneity in iPSC-derived alveolar spheres [42] and fate divergence with some cells capable of generating AT2 cells, while others switch to an alternative non-lung endodermal fate [43].

Organ-on-chip technologies are increasingly powerful in modelling lung stem cell biology. ZAMProgNo and co-workers [44] (University of Bern, Switzerland) presented a new alveolar chip design. This alveolus-on-a-chip improves the ability to model the microenvironment and the complex architecture of the lung parenchyma. Endothelial cells can be co-cultured with patient-derived lung alveolar epithelial cells at the air-liquid interface (ALI) and can be subjected to physiological stretch to mimic breathing. Although the thickness of membranes used in the chips varies from $4.5 \pm 0.8 \mu \mathrm{m}$ to $11.5 \pm 1.2 \mu \mathrm{m}$, thicker than the alveolar wall measures $0.5-0.7 \mu \mathrm{m}$ in vivo [45], this model reproduces some key features of the lung alveolar environment in terms of structure, customisable extracellular matrix (ECM) composition, barrier functions and dynamic microenvironment, providing a promising tool to evaluate interactions between the lung ECM and cells.

\section{Session III. Stem cell niche interactions}

Endogenous lung progenitor cells have a critical and dynamic interaction with their niche. The self-renewal and differentiation of lung progenitor cells are regulated by neighbouring epithelial cells, stromal cells (including fibroblasts, smooth muscle cells and endothelium) and immune cells. Fibroblasts upregulate the expression of interleukin (IL)-6 following injury of the airway epithelium to promote ciliated cell differentiation from basal stem cells in mice [46]. A specific mesenchymal lineage expressing Axin2 and platelet-derived growth factor receptor A (PDGFRA) could provide signals including IL-6, fibroblast growth factors (FGFs) and bone morphogenic protein (BMP) antagonists that promote the self-renewal and differentiation of AT2s [18]. S. Reynolds (Nationwide Children's Hospital, USA) highlighted the role of the niche in lung tissue injury and repair. She posed important questions about the capacity to repair a defective niche and how this can be used to enhance regenerative potential in the human lung. Lung progenitor cells can exit, move, and return to their niche and also create an entirely new niche, underscoring the dynamic interactions between cells and their environment. The critical role of immune cells in the regenerative tissue niche was also highlighted, a topic discussed further in Session VII.

Idiopathic pulmonary fibrosis (IPF) is an intractable interstitial lung disease with poor prognosis. Lung transplantation is often the only therapeutic option for IPF patients. IPF can be divided into sporadic and familiar IPF. Around 30\% of genetic mutations causative for familial IPF have been identified, however a genetic underpinning for sporadic IPF has yet to be discovered. scRNA-seq offers the opportunity to examine cells and cellular interactions between cell populations in regions of fibrosis [47]. Through this, a novel population of pro-fibrotic alveolar macrophages has been discovered exclusively in patients with fibrosis [47]. In a different study a population of $\mathrm{CX} 3 \mathrm{CR} 1^{+}$SiglecF $\mathrm{F}^{+}$transitional macrophages were found to localise to the fibrotic niche and play a key role in driving pro-fibrotic cellular signalling pathways [48]. Age-related mechanisms, including epithelial cellular senescence have been proposed as pathogenic drivers, as the lung alveolar epithelium is gradually obliterated and replaced with nonfunctional fibrotic tissue in IPF. This is proposed to occur due to repetitive injury to the alveolar epithelium and an inability to properly restore function in patients with IPF [49]. LeHmann and co-workers [50] (Comprehensive Pneumology Centre Munich, Germany) showed that there is a loss of alveolar epithelial cells in aged mice. This loss correlates with an elevation of canonical Wnt signalling in the lung epithelium of these mice. Accumulation of active $\beta$-catenin in these cells led to cellular senescence in vitro. In line with recent evidence that IPF is a disease with accelerated ageing of the lung, a fibrotic mouse lung following bleomycin administration and fibrotic human lung tissue had more senescence in AT2 cells as compared 
to healthy controls. AT2 cell senescence may be a mechanism detrimental to lung repair and contributes to the formation of fibrosis. Senolytic drugs, agents that selectively induce apoptosis of senescent cells are capable of clearing senescent cells and reducing fibrotic markers and therefore may be a viable therapeutic option for IPF patients. Studies in bleomycin-induced fibrosis in mice have shown an abundance of senescence biomarkers and the secretome of senescent fibroblasts, that was fibrogenic, could selectively be suppressed by a senolytic cocktail comprising dasatinib plus quercetin alleviating IPF-related dysfunction $[51,52]$. Pilot studies in humans support feasibility of this approach and it will be interesting to follow developments as controlled human trials progress [52].

The niche and tissue of origin can also have important, long lasting impact on cells isolated from these tissues. Y. Yuan (Yale University, USA) presented methods for the purification of pulmonary microvascular endothelial cells from the lower lymphatics of rat lungs (LLPMECs). While morphologically homogeneous, at least six heterogenous endothelial populations were identified in the native pulmonary vasculature by scRNA-seq. The LLPMECs could be used to repopulate the vasculature of decellularised rat lungs, providing a purified cell candidate for regeneration of the pulmonary microvasculature. A combination of purified progenitors with the greatest regenerative potential together with an ECM of native lung morphology were key to this successful tissue regeneration. These sessions further highlighted the importance of understanding precise stem cell and niche interactions for sustained tissue regeneration.

\section{Session IV. Towards the development of new therapies using lung stem cells for cystic fibrosis}

Recent progress has led to the development of drug combinations which can restore CF transmembrane conductance regulator (CFTR) channel function in $\sim 90 \%$ of CF patients; unfortunately $7-10 \%$ of patients do not produce any appreciable amount of CFTR protein (e.g. due to premature stop mutations or aberrant splicing), requiring alternative therapeutic approaches. One option is to correct disease-causing CFTR mutations via editing in a CF patient's own airway basal stem cells. Gene-editing approaches themselves still have a number of limitations to be overcome prior to their in vivo application including avoiding the host immune response, delivery to the desired cell types within the lung, editing with high-efficiency and precision with no off-target effects. Such limitations may be overcome by ex vivo editing and cellular selection; however, ex vivo expansion of lung stem cells comes with its own challenges. GREANEY and co-workers [53] (Yale University, USA) presented data comparing the regenerative potential of rat tracheal basal cells, expanded in vitro using pharmacological expansion techniques, across four different differentiation platforms: organoids, ALI, decellularised trachea and lung. By benchmarking these engineered samples to native rat tracheal and lung epithelial scRNA-seq samples, analogous populations were compared transcriptomically and a population of cells primed for differentiation was identified. Interestingly, the differentiation capacity was heavily dependent upon the model system with even submerged cultures on engineered trachea generating a more robust ciliated epithelium defined by consistent generation of a pseudo-stratified epithelium and mucociliary differentiation. This study highlights the importance of niche and ECM composition on differentiation capacity of basal cells. A significant effort within the lung field is currently being devoted to scRNA-seq analysis of basal cell populations present in the lungs of individuals with $\mathrm{CF}$ and to compare their frequency, their transcriptome and their biological activity with that of basal cells from non-CF lungs. G. Carraro (Cedars-Sinai Medical Center, USA) presented intriguing scRNA-seq data indicating that there are distinct classes of lung basal cells pre-disposed different differentiation fates. It should be noted that such detailed transcriptional characterisation (e.g. cycling status), not only of basal cells, but also other airway epithelial cell types within the CF lung will be invaluable in guiding efforts for in vivo CFTR gene editing.

Another potential source of CFTR-corrected lung stem cells for transplantation, would be to first derive iPSCs from CF patient cells, perform CFTR gene editing and then differentiate to airway basal cells in sufficient quantity for transplantation. CFTR editing has been previously achieved in CF patient-iPSCs which, when subsequently differentiated, exhibited restored CFTR function [54, 55]. J. Mahoney (CF Foundation Therapeutics Laboratory, USA) presented progress toward generating iPSC-derived basal cells utilising a double fluorescent reporter system in which a knock-in NKX2.1-GFP reporter was utilised to read out commitment to lung epithelial fate and a knock-in TP63-tdTomato reporter to identify the development of basal cells. As primary airway basal cells can develop into an airway epithelium at an ALI, iPSC-derived basal cells are also being functionally evaluated in this culture system. Once such differentiation protocols are robust and reproducible, significant work will be required to assess how best to deliver and engraft such cells in the affected human lung.

As we move toward evaluating the in vivo engraftment potential of basal and other airway epithelial progenitor cells, the need for an animal model that reflects disease progression in humans will be essential. LYNCH and co-workers [56, 57] (University of Iowa, USA) presented data on an orthotopic left lung 
transplant model in ferrets, which can also be used to provide a robust model for CF. Ferrets share similarities in lung function and architecture with humans and CF ferrets develop airway obstruction, accumulate thick mucus and develop inflammation. The orthotopic ferret lung transplant model develops the entire spectrum of chronic lung allograft dysfunction pathology, including both obliterative bronchiolitis (OB), characterised by inflammation and irreversible fibrosis obstructing airflow in the small airways [58], and restrictive allograft syndrome (RAS), characterised by a persistent decline in lung capacity due to diffuse alveolar damage and presence of intra-alveolar fibroblasts [59, 60]. Further development and use of larger animal models, such as the ferret, will be pertinent to the robust pre-clinical evaluation of cellular therapies for the human lung.

\section{Session V. Careers in stem cells, cell therapies and lung bioengineering}

Following in the steps of previous conferences, there was a dedicated focus on highlighting the research, mentorship and training of junior investigators in the field of stem cells, bioengineering and cell therapy. This session provided valuable information on academic career support and early career development [5, 6]. There were targeted sessions to discuss issues of gender equity in science, the challenges of starting a laboratory, including the funding and financial management aspects, publishing and reviewing for medical journals and the pros and cons of pre-print servers. Complete details of this session and further resources are provided in the data supplement.

\section{Session VI. Bridging cell and tissue-derived products to market: regulation and commercialisation of regenerative medicine products}

Regenerative medicine is undergoing unprecedented growth as witnessed by the large number of cell- and gene-based therapies (CGTs) currently under evaluation in clinical trials and the over 40 products with marketing authorisation worldwide [61]. The US Food and Drug Administration (FDA) estimates it will receive more than 200 investigational new drug (IND) applications in this area by 2020 and anticipates approving 10-20 CGTs a year by 2025 [62]. This growth can be partly attributed to the introduction of the regenerative medicine advanced therapy designation that simplifies the criteria for expedited clinical testing and allows for multicentre data collection and pooling using one clinical trial protocol [63].

The rapid expansion of the regenerative medicine product pipeline introduces regulatory and manufacturing challenges. Large quantities of good manufacturing practice-compliant cellular products and/or gene vectors will be required for pre-clinical and clinical testing, along with guidance to navigate the new regulatory landscape. A publicly funded initiative to overcome such challenges is the National Heart, Lung and Blood Institute (NHLBI)-funded Production Assistance for Cellular Therapies (PACT) [64]. PACT provides manufacturing support for pre-clinical testing and phase I/II clinical trials, regulatory assistance and streamlines US FDA-compliant data collection. R. LindBLAD, [65, 66] PACT Director, reported that PACT has supported cell products of programmatic interest to the NHLBI, ranging from iPSC-derived cardiomyocytes for acute myocardial infarction to MSCs for acute respiratory distress syndrome (ARDS). In parallel, PACT continues to provide regulatory support through seminars, gap analysis for regulatory compliance and assistance with pre-IND interactions with the US FDA and IND filing.

A regenerative medicine product that exemplifies the enormous clinical potential of CGTs and the long process from basic research to proven therapy is chimeric antigen receptor (CAR)-T-cell gene therapy [67]. CAR-T therapies are a form of adaptive T-cell transfer (ACT) for cancer therapy and present an improvement over other ACTs, due to their major histocompatibility complex-independent target recognition. A CAR is created by combining an antigen recognition domain of antibody with intracellular signalling domains into a single chimeric protein. CARs encoded by lentiviral vectors then stably transduce autologous patient T-cells, which are expanded ex vivo and transferred back to patients. Although the creation of CARs was first described in the 1980s [68, 69] and the first CAR-T clinical trial took place in 1997, it was only recently that CAR-T therapies gained traction, following high remission rates of treated patients and low long-term toxicities. Two CAR-T therapies have obtained marketing authorisation in several jurisdictions (USA, European Union, Switzerland, Australia, Canada and Japan) [61] and are currently under testing in hundreds of clinical trials worldwide. LEvine and co-workers [70] (University of Pennsylvania, USA), co-inventor of CAR-T therapies, detailed challenges remaining for widespread implementation of CAR-T therapies, including variability in patient-derived raw material, expansion of treated conditions and manufacturing shortages for clinical-grade lentiviral vectors.

Another facet of regenerative medicine is the in vitro generation of tissues to ultimately restore organ/ tissue function in patients. Decellularised organ scaffolds have been studied for this purpose but emerging technologies, such as 3D bioprinting, are attractive alternatives. Intense research efforts are directed at developing biomaterials that support various cell types, are compatible with 3D-printing techniques and 
encourage vascularisation. According to M. DeSantis (Lund University, Sweden), hybrid-hydrogels consisting of alginate and decellularised lung ECM meet many of these criteria and have been used as a bioink, combined with human bronchial epithelial cells and extruded into airway-like geometries using the FRESH technique pioneered by FEINBERG and co-workers [71] (Session II). Overall, this session demonstrated the impressive progress in bringing safe and effective CGTs to patients. It also highlighted the challenges ahead, including development of innovative and cost-effective regenerative medicine treatments that enable adoption in clinical practice.

\section{Session VII. Immunomodulation via cell therapy and cell-derived extracellular vesicles}

Clinical translation of MSC-based therapies remains an area of intensive study in respiratory medicine. There are still many questions to be answered about their biology and mechanisms of action to safely harness their full clinical therapeutic potential [72]. Work from THEBAuD's group [5-8] (Ottawa Hospital Research Institute, Canada) is focused on the development of human umbilical cord tissue-derived MSCs for the treatment of bronchopulmonary dysplasia (BPD). Challenges still faced by the cell therapy field include optimisation and standardisation of the cell manufacturing processes, such as: 1) the cell source (e.g. bone marrow, cord tissue, placenta); 2) improved isolation, culture, expansion and cryopreservation techniques; 3) evaluation of cryopreserved versus fresh cellular products; 4) autologous versus allogeneic strategies; and 5) cellular versus cell-free delivery. Several assays were proposed in the session to address a lack of a reliable and rapid disease-specific potency assay that would enable prediction of the therapeutic efficacy of cell therapies. Recent advances in 3D lung organoid culture present a promising approach for evaluating personalised therapeutic interventions on patient-derived material [73-75].

Another exciting research avenue is the recognition that MSCs exert their therapeutic effects through the release of extracellular vesicles (EVs). S. Kourembanas' [76-78] group (Boston Children's Hospital, USA) has been investigating the therapeutic potential of MSC-derived exosomes (MEx) (the smaller-sized EV fraction that contains proteins and miRNA). Their work has demonstrated that MEx improve histological and functional outcomes in experimental models of pulmonary hypertension, BPD and lung fibrosis. Interestingly, MEx treatment modulated lung and bone marrow monocyte populations towards an anti-inflammatory M2-like phenotype [77]. MEx-based therapeutics represent a promising innovative approach to treat lung diseases without the safety concerns associated with live cell treatments. Data presented by J. Dutra Silva (Queen's University Belfast, UK) indicated the potential for MSC EVs to improve epithelial-endothelial barrier functions in pre-clinical models of ARDS. This work highlighted the importance of functional mitochondria in the EV preparations and further emphasised the importance of proper characterisation and standardisation of the cell products and understanding the mechanisms of their therapeutic effects. Elucidation of the functional role of the specific EV contents (e.g. miRNAs, proteins, mitochondria) may lead to identification of novel therapeutic targets. A certain caution should be excised as recent studies using xenograft tumour models have suggested that MSC EVs may be associated with increased proliferation and decreases apoptosis in lung adenocarcinoma cancer cells. The mechanism was demonstrated to be via EV-transmitted miR-410 and suggests that modifications of MSC-EV may be essential to decrease unwanted therapeutic side effects [79].

J. Collins (Erasmus University Medical Center, Netherlands), discussed the involvement of the endogenous lung MSCs in pathological changes leading to BPD and the role of microbiome in shaping endogenous MSC populations. Indeed, accumulating evidence suggests that disease microenvironment can influence MSC behaviours and consequently therapeutic potential $[9,80,81]$. Therefore, a better understanding of the lung injury microenvironment and how it alters functional properties of the cell-based therapies is essential to their effective clinical translation and will be an important area of further investigation.

\section{Session IX. New strategies for cellular delivery and regenerative medicine}

Understanding how cells or cellular derivatives, such as EVs or ECM, influence cell behaviour in the lung is central to designing cellular therapies for acute and chronic lung diseases. As previously discussed, MSCs have potential therapeutic benefit in the treatment of several lung diseases [5, 9, 72]. PATI and co-workers [82, 83] (University of California San Francisco, USA) has shown that MSC actions are mediated by different effectors in different diseases. In the context of traumatic brain injury, for example, TIMP3 was identified as a mediator of MSC function; however, in acute lung injury, the positive effects of MSCs are TIMP3 independent. Similarly, while some of the MSCs effects can be phenocopied by using MSC exosomes, others appear to be cell mediated. In addition, some of the effects observed in vivo could not be recapitulated in vitro, suggesting the involvement of an intermediate cell. Understanding of these and other mechanisms involved in the beneficial effects of MSCs are needed to further tailor treatment efficacy and safety to the specific patient and disease. 
Chronic lung allograft dysfunction presents as RAS and OB and limits long-term survival following lung transplant. Recently, a depletion of endogenous lung basal stem cells was described, limiting repair in these situations. The mechanisms driving stem cell depletion remain a major challenge in the field. Using a ferret model of OB, PAREKH and co-workers [84] (University of Iowa, USA) demonstrated that both small and large airways are affected in $\mathrm{OB}$, a disease previously believed to affect only distal airways. Upon $\mathrm{OB}$ development, basal epithelial cells are diminished from small and large airways and submucosal glands. Delivery of ex vivo expanded lung basal stem cells showed promising results regenerating surface airway epithelium, suggesting that application of basal lung stem cells upon lung transplantation may offset the disruption of endogenous airway stem cell niches and prolong patient survival.

An alternate approach to donor lung transplantation is the generation of bioartificial lungs by 3D bioprinting. The development of 3D-printable bioinks from extracellular matrix is a promising approach that harnesses the potential of the ECM to instruct cell behaviour [85]. An example of this was presented by GALLIGER and co-workers [85] (University of Minnesota, USA) who use ECM derived from porcine trachea to develop bioinks that are capable of inducing neocartilage production in MSCs. Different extraction techniques were analysed for their effects if incorporated into hydrogels. A urea-based extraction improves the compressive modulus of the final 3D bioprinted tracheal constructs and allows for cartilage differentiation and collagen II production by incorporated human MSCs. Further investigations into cellular remodelling in long-term cultures are warranted, exploring functional properties of the artificial cartilage.

\section{Session $\mathrm{X}$. Visualising lung regeneration}

The adult lung is a relatively quiescent organ; however, upon injury, different regional-specific stem and progenitor cells interact with tissue-resident and recruited immune cells to orchestrate repair. However, mechanisms underlying such regeneration (and its dysregulation in disease) are still poorly understood [86, 87]. Pioneers, such as WeIBEL and co-workers [88], paved the way for using imaging techniques to advance our understanding of lung regeneration. Early studies relied mostly on static 2D imaging methods, such as electron microscopy and histology, which are not suitable for live imaging and therefore limited in their ability to visualise lung regeneration. The lung is a particularly difficult organ to image at high cell and tissue resolution because it is shielded by the thoracic cage, filled with air creating artefacts at the air-tissue interface, and is a dynamic organ. One approach to visualise cell interactions and/or differentiation in a more in vivo like environment is to isolate and culture cells in 3D scaffolds (synthetic or naturally derived) and image regenerative processes overtime. M. Ho (Ottawa Hospital Research Institute, Canada) established a novel co-culture model of iPSC-derived smooth muscle cells (iSMCs) from healthy patients or those with lymphangioleiomyomatosis (LAM) combined with endothelial cells (ECs) in Matrigel to examine the role of this cellular crosstalk in endothelial tube formation. Co-culture of normal ECs with LAM iSMCs resulted in EC network degradation and apoptosis, which was not seen for iSMCs derived from healthy patients (unpublished data).

Using co-culture models has many advantages, such as control over cellular complexity and the ability to use human cells, but does not fully represent the complexity of native tissue. Although technically challenging, in situ or ex vivo imaging of the lung is a more ideal scenario for visualising lung regeneration. One recent example of the power of this approach is the study by Li et al. [89], in which both in situ and ex vivo imaging of murine fetal lungs was used to explore the link between mechanical and chemical cues in lung development. During the session, Gusarova [90, 91] (Columbia University, USA) highlighted recent technical advances for visualisation of acute lung injury and repair in ex vivo, blood-perfused mouse lungs using live confocal microscopy imaging. This setup allows for administration of drugs and other compounds during imaging; however, the system still lacks movements associated with normal breathing. Alveoli could be visualised at high magnification to give insights insight into mechanisms of injury and repair in the alveolar epithelium, such as the dynamic regulation of proinflammatory receptors by the cytosolic calcium and cellular actin. An emerging alternative for high resolution optical imaging of native lung tissue is the use of PCLSs. PCLSs contain most native cell types and retain the tissue architecture and ECM of the native lung [92]. Furthermore, PCLSs can be generated from human tissue and there are currently no other techniques that allow for the visualisation of human lung tissue over time at this resolution. C. Dean (Imperial College London, UK), presented her group's recent work using PCLSs from post-natal mice to visualise alveologenesis. Using a bright-field fluorescent microscope-based setup, they were able to image the lung tissue with high spatiotemporal resolution and observed several key stages of alveolar formation: 1) highly mobile epithelial cells, 2) cell clustering, and 3) hollowing [93]. While confocal and conventional fluorescence microscopy allow for imaging lung regeneration at high resolution, it is hampered by difficulties in penetrating thick tissue samples, long imaging acquisition times and phototoxicity. One emerging imaging technique that overcomes many of these limitations is light-sheet microscopy, which was recently used to visualise nanoparticle deposition in 
TABLE 1 Conference summary, recommendations and focus areas. Many of the recommendations from our previous conference remain valid with priorities for research focus and funding recommendations [95]. The current table comprises new recommendations arising specifically from the 2019 conference

\section{Basic Science: Analysis and Visualisation of Endogenous Lung Stem Cells}

- Continue advancement of the utility of scRNA-seq through 3D visualisation of the datasets ("spatialomics") in conjunction with spatial transcriptomics and application of the technology and data integration across multiple species.

- Continue progression in applying a systems-level approach to discover interactions through mining of scRNA-seq data to identify fundamental cell signalling pathways and networks in homeostasis and disease.

- Identification of additional cell surface markers that characterise lung cell populations for use in visualisation and sorting techniques through evaluation of current datasets in the human lung.

- Improve access to bioinformaticians with a biological background/understanding of lung disease was a limiting factor in the use of scRNA-seq. Efforts should be made to increase awareness of available courses to aid young scientists in building a background in bioinformatics (e.g. those hosted by the National Bioinformatics Infrastructure in Sweden/SciLife and MIT/Broad Institute in the USA).

- Encourage incorporation of virtual reality technology as a core facility within universities to facilitate worldwide collaborations using interactive data evaluation.

- Improve availability of funding for core services or the development of a cryopreserved biobank for PCLSs to be an available resource for all lung researchers. Suggestion to develop a collaborative RFA.

- Establish techniques for light-sheet microscopy, including live imaging and imaging in human tissue. The limited amount of techniques which can be used to image lung regeneration at high spatiotemporal resolution was reiterated throughout the workshop. Current techniques are limited by difficulties in penetrating thick tissue samples, long imaging acquisition times and phototoxicity.

Basic Science: Induced Pluripotent Stem Cells and Disease Modelling

- Refine and functionally validate protocols to specify lung cells from pluripotent stem cells (iPSC and ESC). Critical comparisons to primary lung cells.

- Continue to develop disease-specific populations of ESC-/iPSC-derived cells, to study rare lung diseases such as cystic fibrosis and $\alpha_{1}$-antitrypsin deficiency.

- Continue to development of high-throughput cellular models from pluripotent sources for screening of novel therapeutics.

- Comprehensively evaluate the effect of environmental influences, including mechanical forces, extracellular matrix, inflammation and infection on development of lung tissue from stem and progenitor cells.

Basic Science: Understanding of the Impact of Stem Cell: Niche Interactions

- Continue to elucidate how endogenous lung stem/progenitor cells are regulated in normal development and during tissue homeostasis.

- Analysis of epigenetic modulation of lung stem cells and how cellular microenvironments change this.

- Understand how autologous iPSC-derived lung lineages will behave in the diseased microenvironment in vivo.

Basic Science: Bioengineering and the Lung

- Continue to explore lung tissue bioengineering approaches such as artificial matrices, 3D culture systems le.g. extracellular matrix environments for organoid culture), 3D bioprinting and other novel approaches for generating lung ex vivo and in vivo from stem cells, including systems that facilitate vascular development.

- Evaluate effect of environmental influences, including oxygen tension and mechanical forces, including stretch and compression pressure, on development of lung tissue from stem and progenitor cells.

- Define the consensus end-points for functional evaluation and validation of engineered lung tissue.

- Continue to improve technology to visualise lung regeneration utilising 3D matrices and PCLSs.

Translational Science: Cell Therapy - Delivery of Stem Cells to the Lung

- Invest in developing larger animal models, such as the ferret and the pig, which have lung structure and function more akin to that of humans, as pre-clinical models to evaluate cellular therapy.

3D: three-dimensional; ESC: embryonic stem cells; iPSC: induced pluripotent stem cells; MIT: Massachusetts Institute of Technology; PCLS: precision-cut lung slice; RFA: request for applications; scRNA-seq: single-cell ribonucleic acid sequencing.

entire mouse lungs ex vivo [94]. Establishment of techniques for light-sheet microscopy, including live imaging and imaging in human tissue, will be important for future studies in lung regeneration.

\section{Setting priorities and recommendations regarding funding future research}

The broad field of lung regenerative medicine continues to evolve at an accelerating pace. The National Institutes of Health (NIH), nonprofit respiratory disease foundations and other sources of scientific and funding support remain positive and this will be important for continued development. As in past conference reports, series of scientific and funding recommendations coming out of discussions at the conference and post-conference surveys are presented in table 1 . This continues to evolve and reflect a growing interest in application of bioengineering approaches and technologies to the study of lung biology and diseases, something highly evident at the 2019 conference. In the final session, we conducted a series of survey questions addressing key theme areas of the conference; advances in biotechnology and 

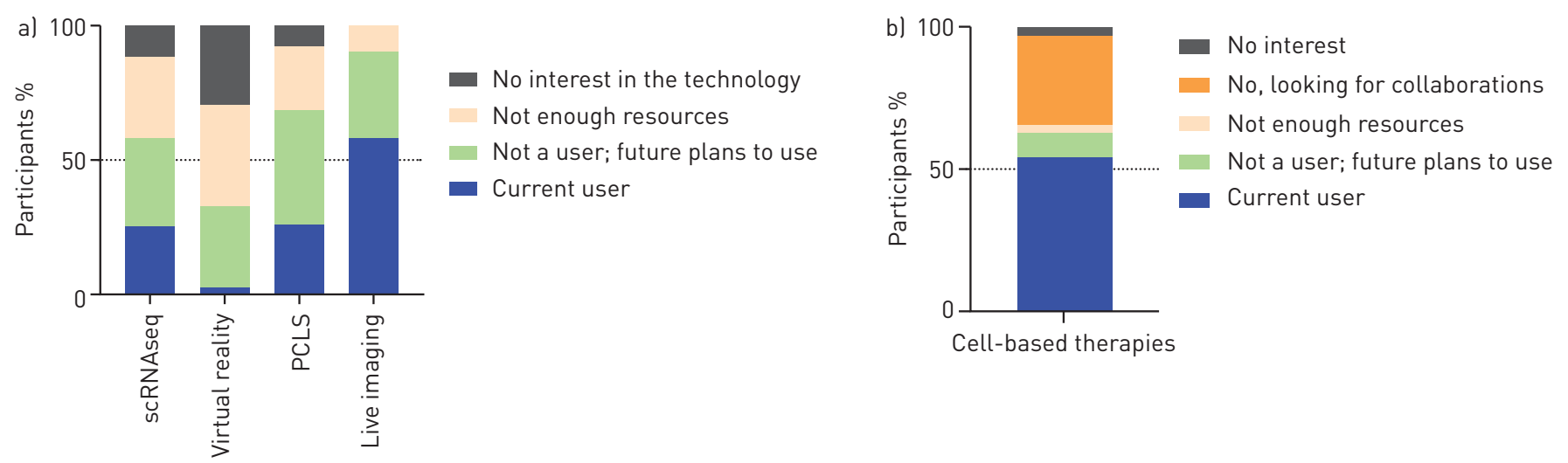

c)

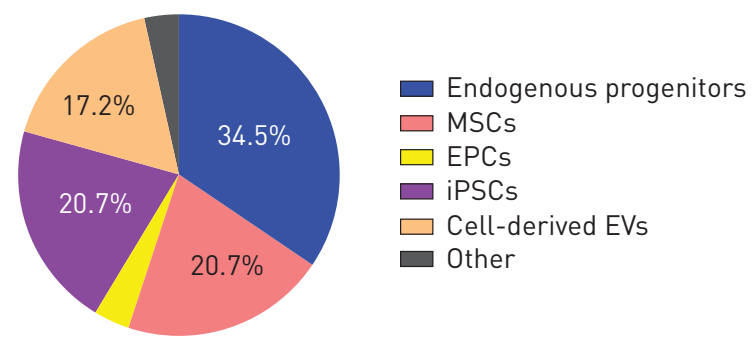

- No interest in the technology

- Not enough resources

- Not a user; future plans to use

Current user

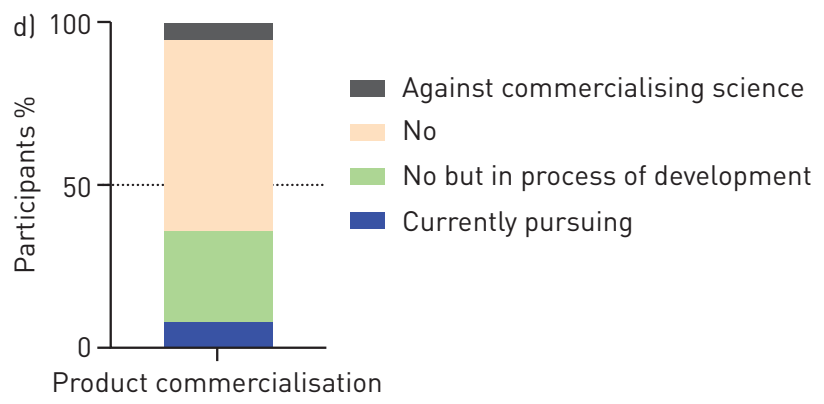

FIGURE 2 Summarised responses to survey questions addressing key theme areas of the conference. Key themes included were advances in biotechnology and bioengineering (a), endogenous lung progenitor cells (b), cell-based therapies (c) and commercialisation (d). scRNA-seq: single-cell ribonucleic acid sequencing; PCLS: precision-cut lung slice; MSC: mesenchymal stromal cell; EPC: epithelial progenitor cell; iPSC: induced pluripotent stem cell.

bioengineering, endogenous lung progenitor cells, cell-based therapies and commercialisation. An audience response system using individual voting remotes was utilised for immediate anonymous responses from participants of the conference (supplemental table 1) to guide discussion and crafting of the overall recommendations

In brief, the survey indicated large and increasing numbers of individuals and research groups utilising technologies such as scRNA-seq and PCLS as well as keen interest in other cutting-edge technologies such as VR (figure 2a). Overall, 30\% of respondents indicated a need for more bioengineering programs, whereas $13 \%$ felt that the current number is sufficient (figure $2 \mathrm{~b}$ ). $94 \%$ of survey responses indicate active, planned, or desired activities in cell-based therapies with the majority indicating interest/activity in endogenous progenitor cells (34\%). However, robust and similar interest in MSCs, iPSCs and EVs highlighted the support for a broad range of approaches (figure 2c). Importantly, there was strong uniform sentiment that these areas are underfunded with a strong call (94\%) for all funding agencies to increase support for cell-based therapies. With respect to commercialisation, $36 \%$ of respondents indicated either a commercialised product or one in development with $87 \%$ indicating adequate support for commercialisation through technology transfer offices or other avenues at their respective institution (figure 2d). Only a small number (9\%) indicated that they required assistance in developing commercialisation strategies. Overall, $72 \%$ of respondents indicated that all funding agencies must increase support for commercialisation pathways. The vast majority of participants indicating plans to participate in the next conference in 2021.

\section{Conclusions}

Since the last conference in 2017, substantial advances in the development of new technologies, increasing utilisation of scRNA-seq data, and the further development of more physiologically relevant 3D cellular models have driven considerable progress in the field moving closer to the development of potential therapeutic strategies for lung repair after injury or in end-stage lung disease. Despite the progress, there are still considerable deficits in our understanding of how transcriptomic changes in different endogenous and derived stem and progenitor cell populations correlate to function at the tissue and organ level, how to describe the stem cell niche and how changes in this niche lead to functional alterations in the these cells. The pursuit of understanding the function of human lung across the spectrum from a subcellular to organ level to improve success of therapeutic translation of our findings will be increasingly dependent on 
collaborative and interdisciplinary research teams. This includes further synthesis and integration of technologic advances highlighted in this conference and elsewhere. This conference continues to bring together leaders across such multidisciplinary areas of active research from basic biologists, bioengineers and bioinformaticians to ethicists, business leaders and policy makers and retains a focus on the promotion of innovation among junior scientists across the world. The recommendations in table 1 compiled from active discussion throughout the conference should provide a platform for stimulating active new research that will progress the field toward its goals for restoration of a functional lung in patients living with acute and chronic lung disease. Each of these recommendations is worthy of intensive and extensive further development and review beyond the scope of this summary.

Conflict of interest: D.E. Wagner reports a grant from the US NIH (R13 HL149436, conference grant), and grants for conference support from the Alpha-1 Foundation, the International Society for Cell and Gene Therapy, and the Pulmonary Fibrosis Foundation, during the conduct of the study; and honoraria from Boehringer Ingelheim outside the submitted work. In addition, D.E. Wagner has patent US20160067378A1 pending. L. Ikonomou has nothing to disclose. S.E. Gilpin has nothing to disclose. C.M. Magin reports consulting fees from Sharklet Technologies, Inc., outside the submitted work; and a patent, "3D in vitro models of lung tissue", pending. F. Cruz has nothing to disclose. A. Greaney has nothing to disclose. M. Magnusson has nothing to disclose. Y-W. Chen has nothing to disclose. B. Davis has nothing to disclose. K. Vanuytsel has nothing to disclose. S. Rolandsson Enes has nothing to disclose. A. Krasnodembskaya reports grants from the Medical Research Council (MRC) UK during the writing of this article. She is funded by the MRC (national funder for medical and translational research) to conduct research in the area of stem cell-based therapies for lung diseases. M. Lehmann has nothing to disclose. G. Westergren-Thorsson has nothing to disclose. J. Stegmayr has nothing to disclose. H.N. Alsafadi has nothing to disclose. E.T. Hoffman has nothing to disclose. D.J. Weiss reports grants from the NIH during the conduct of the study. A.L. Ryan reports grants from the Cystic Fibrosis Foundation and the NIH, outside the submitted work.

Support statement: The organisers thank the NIH/NHLBI (grant R13 HL149436), the Alpha-1 Foundation, the Cystic Fibrosis Foundation, the International Society for Cell and Gene Therapy, the Pulmonary Fibrosis Foundation and the University of Vermont Larner College of Medicine, as well as ACell, Athersys and United Therapeutics. Funding information for this article has been deposited with the Crossref Funder Registry.

\section{References}

1 Legetth $\mathrm{O}$, Rodhe J, Pålsson J, et al. CellexalVR: a virtual reality platform for the visualisation and analysis of single-cell gene expression data. BioRxiv 2019; preprint [https://oi.org/10.1101/329102].

2 Raredon MSB, Adams TS, Suhail Y, et al. Single-cell connectomic analysis of adult mammalian lungs. Sci $A d v$ 2019; 5: eaaw3851.

3 Sachs N, Papaspyropoulos A, Zomer-van Ommen DD, et al. Long-term expanding human airway organoids for disease modeling. $E M B O J$ 2019; 38: e100300.

4 Zacharias WJ, Frank DB, Zepp JA, et al. Regeneration of the lung alveolus by an evolutionarily conserved epithelial progenitor. Nature 2018; 555: 251-255.

5 Augustine S, Avey MT, Harrison B, et al. Mesenchymal stromal cell therapy in bronchopulmonary dysplasia: systematic review and meta-analysis of preclinical studies. Stem Cells Transl Med 2017; 6: 2079-2093.

6 Thebaud B. Mesenchymal stromal cell therapy for respiratory complications of extreme prematurity. Am J Perinatol 2018; 35: 566-569.

7 Boregowda SV, Krishnappa V, Haga CL, et al. A clinical indications prediction scale based on TWIST1 for human mesenchymal stem cells. EBioMedicine 2016; 4: 62-73.

8 Thebaud B. Stem cells for extreme prematurity. Am J Perinatol 2019; 36: S68-S73.

9 Islam D, Huang Y, Fanelli V, et al. Identification and modulation of microenvironment is crucial for effective mesenchymal stromal cell therapy in acute lung injury. Am J Respir Crit Care Med 2019; 199: 1214-1224.

10 Lysaght T, Lipworth W, Hendl T, et al. The deadly business of an unregulated global stem cell industry. J Med Ethics 2017; 43: 744-746.

11 Bauer G, Elsallab M, Abou-El-Enein M. Concise review: a comprehensive analysis of reported adverse events in patients receiving unproven stem cell-based interventions. Stem Cells Transl Med 2018; 7: 676-685.

12 Dominici M, Nichols KM, Levine AD, et al. Science, ethics and communication remain essential for the success of cell-based therapies. Brain Circ 2016; 2: 146-151.

13 Wagner DE, Turner L, Panoskaltsis-Mortari A, et al. Co-opting of ClinicalTrials.gov by patient-funded studies. Lancet Respir Med 2018; 6: 579-581.

14 Tang F, Barbacioru C, Wang Y, et al. mRNA-seq whole-transcriptome analysis of a single cell. Nat Methods 2009; 6: $377-382$.

15 Montoro DT, Haber AL, Biton M, et al. A revised airway epithelial hierarchy includes CFTR-expressing ionocytes. Nature 2018; 560: 319-324.

16 Plasschaert LW, Zilionis R, Choo-Wing R, et al. A single-cell atlas of the airway epithelium reveals the CFTR-rich pulmonary ionocyte. Nature 2018; 560: 377-381.

17 Guo MZ, Du YN, Gokey JJ, et al. Single-cell RNA analysis identifies cellular heterogeneity and adaptive responses of the lung at birth. Nat Commun 2019; 10: 37.

18 Zepp JA, Zacharias WJ, Frank DB, et al. Distinct mesenchymal lineages and niches promote epithelial self-renewal and myofibrogenesis in the lung. Cell 2017; 170: 1134-1148.

19 Lee JH, Tammela T, Hofree M, et al. Anatomically and functionally distinct lung mesenchymal populations marked by Lgr5 and Lgr6. Cell 2017; 170: 1149-1163.

20 Frank DB, Penkala IJ, Zepp JA, et al. Early lineage specification defines alveolar epithelial ontogeny in the murine lung. Proc Natl Acad Sci USA 2019; 116: 4362-4371.

21 Reyfman PA, Walter JM, Joshi N, et al. Single-cell transcriptomic analysis of human lung provides insights into the pathobiology of pulmonary fibrosis. Am J Resp Crit Care 2019; 199: 1517-1536. 
22 Xu Y, Mizuno T, Sridharan A, et al. Single-cell RNA sequencing identifies diverse roles of epithelial cells in idiopathic pulmonary fibrosis. Jci Insight 2016; 1; e90558.

23 Leigh ND, Dunlap GS, Johnson K, et al. Transcriptomic landscape of the blastema niche in regenerating adult axolotl limbs at single-cell resolution. Nat Commun 2018; 9: 5153.

24 Trapnell C, Cacchiarelli D, Grimsby J, et al. The dynamics and regulators of cell fate decisions are revealed by pseudotemporal ordering of single cells. Nat Biotechnol 2014; 32: 381-386.

25 Bendall SC, Davis KL, Amir ED, et al. Single-cell trajectory detection uncovers progression and regulatory coordination in human b cell development. Cell 2014; 157: 714-725.

26 Saelens W, Cannoodt R, Todorov $\mathrm{H}$, et al. A comparison of single-cell trajectory inference methods. Nat Biotechnol 2019; 37: 547-554.

27 La Manno G, Soldatov R, Zeisel A, et al. RNA velocity of single cells. Nature 2018; 560: 494-498.

28 Fan Z, Chen R, Chen X. SpatialDB: a database for spatially resolved transcriptomes. Nucleic Acids Res 2020; 48: D233-D237.

29 Xia C, Fan J, Emanuel G, et al. Spatial transcriptome profiling by MERFISH reveals subcellular RNA compartmentalization and cell cycle-dependent gene expression. Proc Natl Acad Sci USA 2019; 116: 19490-19499.

30 Chen KH, Boettiger AN, Moffitt JR, et al. RNA imaging. Spatially resolved, highly multiplexed RNA profiling in single cells. Science 2015; 348: aaa6090.

31 Nichterwitz S, Benitez JA, Hoogstraaten R, et al. LCM-seq: a method for spatial transcriptomic profiling using laser capture microdissection coupled with polyA-based RNA sequencing. Methods Mol Biol 2018; 1649: 95-110.

32 Nichterwitz S, Chen G, Aguila Benitez J, et al. Laser capture microscopy coupled with Smart-seq2 for precise spatial transcriptomic profiling. Nat Commun 2016; 7: 12139.

33 Liu Y, Yang M, Deng Y, et al. High-spatial-resolution multi-omics atlas sequencing of mouse embryos via deterministic barcoding in tissue. BioRxiv 2019; preprint [https://doi.org/10.1101/788992].

34 McDole K, Guignard L, Amat F, et al. In toto imaging and reconstruction of post-implantation mouse development at the single-cell level. Cell 2018; 175: 859-876.

35 Hinton TJ, Jallerat Q, Palchesko RN, et al. Three-dimensional printing of complex biological structures by freeform reversible embedding of suspended hydrogels. Sci Adv 2015; 1: e1500758.

36 Lee A, Hudson AR, Shiwarski DJ, et al. 3D bioprinting of collagen to rebuild components of the human heart Science 2019; 365: 482-487.

37 Gotoh S, Ito I, Nagasaki T, et al. Generation of alveolar epithelial spheroids via isolated progenitor cells from human pluripotent stem cells. Stem Cell Reports 2014; 3: 394-403.

38 Konishi S, Gotoh S, Tateishi K, et al. Directed induction of functional multi-ciliated cells in proximal airway epithelial spheroids from human pluripotent stem cells. Stem Cell Reports 2016; 6: 18-25.

39 Korogi Y, Gotoh S, Ikeo S, et al. In vitro disease modeling of Hermansky-Pudlak syndrome type 2 using human induced pluripotent stem cell-derived alveolar organoids. Stem Cell Reports 2019; 13: 235.

40 Jacob A, Morley M, Hawkins F, et al. Differentiation of human pluripotent stem cells into functional lung alveolar epithelial cells. Cell Stem Cell 2017; 21: 472-488.

41 Jacob A, Vedaie M, Roberts DA, et al. Derivation of self-renewing lung alveolar epithelial type II cells from human pluripotent stem cells. Nat Protoc 2019; 14: 3303-3332.

42 McCauley KB, Alysandratos KD, Jacob A, et al. Single-cell transcriptomic profiling of pluripotent stem cell-derived SCGB3A2 ${ }^{+}$airway epithelium. Stem Cell Reports 2018; 10: 1579-1595.

43 Hurley K, Ding J, Villacorta-Martin C, et al. Reconstructed single-cell fate trajectories define lineage plasticity windows during differentiation of human PSC-derived distal lung progenitors. Cell Stem Cell 2020; 26: 593-608 e598.

44 Zamprogno $\mathrm{P}$, Wüthrich S, Achenbach S, et al. Second-generation lung-on-a-chip array with a stretchable biological membrane. BioRxiv 2019; preprint [https://doi.org/10.1101/608919].

45 Papadakos P, Lachmann B. Mechanical ventilation: clinical applications and pathophysiology. Philadelphia Saunders, 2007.

46 Tadokoro T, Wang Y, Barak LS, et al. IL-6/STAT3 promotes regeneration of airway ciliated cells from basal stem cells. Proc Natl Acad Sci USA 2014; 111: E3641-E3649.

47 Reyfman PA, Walter JM, Joshi N, et al. Single-cell transcriptomic analysis of human lung provides insights into the pathobiology of pulmonary fibrosis. Am J Respir Crit Care Med 2019; 199: 1517-1536.

48 Aran D, Looney AP, Liu L, et al. Reference-based analysis of lung single-cell sequencing reveals a transitional profibrotic macrophage. Nat Immunol 2019; 20: 163-172.

49 Datta A, Scotton CJ, Chambers RC. Novel therapeutic approaches for pulmonary fibrosis. Br J Pharmacol 2011, 163: $141-172$.

50 Lehmann M, Korfei M, Mutze K, et al. Senolytic drugs target alveolar epithelial cell function and attenuate experimental lung fibrosis ex vivo. Eur Respir J 2017; 50: 1602367.

51 Schafer MJ, White TA, Iijima K, et al. Cellular senescence mediates fibrotic pulmonary disease. Nat Commun 2017; 8: 14532

52 Justice JN, Nambiar AM, Tchkonia T, et al. Senolytics in idiopathic pulmonary fibrosis: results from a first-in-human, open-label, pilot study. EBioMedicine 2019; 40: 554-563.

53 Greaney AM, Adams TS, Brickman Raredon MS, et al. Platform effects on regeneration by pulmonary basal cells as evaluated by single-cell RNA sequencing. Cell Rep 2020; 30: 4250-4265.

54 Firth AL, Menon T, Parker GS, et al. Functional gene correction for cystic fibrosis in lung epithelial cells generated from patient iPSCs. Cell Rep 2015; 12: 1385-1390.

55 Crane AM, Kramer P, Bui JH, et al. Targeted correction and restored function of the CFTR gene in cystic fibrosis induced pluripotent stem cells. Stem Cell Reports 2015; 4: 569-577.

56 Sun X, Sui H, Fisher JT, et al. Disease phenotype of a ferret CFTR-knockout model of cystic fibrosis. J Clin Invest 2010; 120: 3149-3160

57 Li Z, Sun X, Chen J, et al. Cloned ferrets produced by somatic cell nuclear transfer. Dev Biol 2006; 293: 439-448.

58 Krishna R, Oliver TI. Bronchiolitis Obliterans (Obliterative Bronchiolitis, Constrictive Bronchiolitis). Treasure Island (FL), StatPearls, 2020. 
59 Glanville AR, Verleden GM, Todd JL, et al. Chronic lung allograft dysfunction: definition and update of restrictive allograft syndrome. A consensus report from the Pulmonary Council of the ISHLT. J Heart Lung Transplant 2019; 38: $483-492$

60 Traxler D, Schweiger T, Schwarz S, et al. The lymphatic phenotype of lung allografts in patients with bronchiolitis obliterans syndrome and restrictive allograft syndrome. Transplantation 2017; 101:310-315.

61 Cuende N, Rasko JEJ, Koh MBC, et al. Cell, tissue and gene products with marketing authorization in 2018 worldwide. Cytotherapy 2018; 20: 1401-1413.

62 US Food and Drug Administration. Statement from FDA Commissioner Scott Gottlieb, M.D. and Peter Marks, M.D., Ph.D., Director of the Center for Biologics Evaluation and Research on new policies to advance development of safe and effective cell and gene therapies 2019. www.fda.gov/news-events/press-announcements/ statement-fda-commissioner-scott-gottlieb-md-and-peter-marks-md-phd-director-center-biologics Date last accessed: 11 September 2019. Date last updated: 15 January 2019.

63 Marks P, Gottlieb S. Balancing safety and innovation for cell-based regenerative medicine. N Engl J Med 2018; 378: 954-959.

64 Wood D, Wesselschmidt R, Hematti P, et al. An update from the United States National Heart, Lung, and Blood Institute- funded Production Assistance for Cellular Therapies (PACT) Program: a decade of cell therapy. Clin Transl Sci 2014; 7: 93-99.

65 Liu YW, Chen B, Yang XL, et al. Human embryonic stem cell-derived cardiomyocytes restore function in infarcted hearts of non-human primates. Nat Biotechnol 2018; 36: 597-605.

66 Matthay MA, Calfee CS, Zhuo HJ, et al. Treatment with allogeneic mesenchymal stromal cells for moderate to severe acute respiratory distress syndrome (START study): a randomised phase 2a safety trial. Lancet Resp Med 2019; 7: 154-162.

67 June CH, O'Connor RS, Kawalekar OU, et al. CAR T cell immunotherapy for human cancer. Science 2018; 359: $1361-1365$.

68 Kuwana Y, Asakura Y, Utsunomiya N, et al. Expression of chimeric receptor composed of immunoglobulin-derived v-regions and T-cell receptor-derived c-regions. Biochem Biophys Res Commun 1987; 149: $960-968$.

69 Gross G, Waks T, Eshhar Z. Expression of immunoglobulin-T-cell receptor chimeric molecules as functional receptors with antibody-type specificity. Proc Natl Acad Sci USA 1989; 86: 10024-10028.

70 Roddie C, O’Reilly M, Pinto JDA, et al. Manufacturing chimeric antigen receptor T cells: issues and challenges. Cytotherapy 2019; 21: 327-340.

71 Hinton TJ, Jallerat Q, Palchesko RN, et al. Three-dimensional printing of complex biological structures by freeform reversible embedding of suspended hydrogels. Sci Adv 2015; 1: 10.

72 Galipeau J, Sensebe L. Mesenchymal stromal cells: clinical challenges and therapeutic opportunities. Cell Stem Cell 2018; 22: 824-833.

73 Miller AJ, Dye BR, Ferrer-Torres D, et al. Generation of lung organoids from human pluripotent stem cells in vitro. Nat Protoc 2019; 14: 518-540.

74 Sucre JMS, Jetter CS, Loomans H, et al. Successful establishment of primary type ii alveolar epithelium with 3D organotypic coculture. Am J Respir Cell Mol Biol 2018; 59: 158-166.

75 Tan Q, Choi KM, Sicard D, et al. Human airway organoid engineering as a step toward lung regeneration and disease modeling. Biomaterials 2017; 113: 118-132.

76 Lee C, Mitsialis SA, Aslam M, et al. Exosomes mediate the cytoprotective action of mesenchymal stromal cells on hypoxia-induced pulmonary hypertension. Circulation 2012; 126: 2601-2611.

77 Willis GR, Fernandez-Gonzalez A, Anastas J, et al. Mesenchymal stromal cell exosomes ameliorate experimental bronchopulmonary dysplasia and restore lung function through macrophage immunomodulation. Am J Respir Crit Care Med 2018; 197: 104-116.

78 Willis GR, Mitsialis SA, Kourembanas S. "Good things come in small packages": application of exosome-based therapeutics in neonatal lung injury. Pediatr Res 2018; 83: 298-307.

79 Dong L, Pu Y, Zhang L, et al. Human umbilical cord mesenchymal stem cell-derived extracellular vesicles promote lung adenocarcinoma growth by transferring miR-410. Cell Death Dis 2018; 9: 218 .

80 Weiss DJ, English K, Krasnodembskaya A, et al. The necrobiology of mesenchymal stromal cells affects therapeutic efficacy. Front Immunol 2019; 10: 1228.

81 Gupta N, Sinha R, Krasnodembskaya A, et al. The TLR4-PAR1 axis regulates bone marrow mesenchymal stromal cell survival and therapeutic capacity in experimental bacterial pneumonia. Stem Cells 2018; 36: 796-806.

82 Menge T, Zhao Y, Zhao J, et al. Mesenchymal stem cells regulate blood-brain barrier integrity through TIMP3 release after traumatic brain injury. Sci Transl Med 2012; 4: 161ra150.

83 Pati S, Gerber MH, Menge TD, et al. Bone marrow derived mesenchymal stem cells inhibit inflammation and preserve vascular endothelial integrity in the lungs after hemorrhagic shock. PLoS ONE 2011; 6: e25171.

84 Swatek AM, Lynch TJ, Crooke AK, et al. Depletion of airway submucosal glands and TP63( $\left(^{+}\right) \mathrm{KRT} 5\left(^{+}\right)$basal cells in obliterative bronchiolitis. Am J Respir Crit Care Med 2018; 197: 1045-1057.

85 Galliger Z, Panoskaltsis-Mortari A. Tracheal cartilage isolation and decellularization. Methods Mol Biol 2018; 1577 $155-160$

86 Kotton DN, Morrisey EE. Lung regeneration: mechanisms, applications and emerging stem cell populations. Nat Med 2014; 20: 822-832.

87 Beers MF, Morrisey EE. The three R's of lung health and disease: repair, remodeling, and regeneration. J Clin Invest 2011; 121: 2065-2073.

88 Kaplan HP, Robinson FR, Kapanci Y, et al. Pathogenesis and reversibility of the pulmonary lesions of oxygen toxicity in monkeys. I. Clinical and light microscopic studies. Lab Invest 1969; 20: 94-100.

$89 \mathrm{Li} \mathrm{J}$, Wang Z, Chu Q, et al. The strength of mechanical forces determines the differentiation of alveolar epithelial cells. Dev Cell 2018; 44: 297-312.

90 Rowlands DJ, Islam MN, Das SR, et al. Activation of TNFR1 ectodomain shedding by mitochondrial Ca2 determines the severity of inflammation in mouse lung microvessels. J Clin Invest 2011; 121: 1986-1999.

91 Hough RF, Islam MN, Gusarova GA, et al. Endothelial mitochondria determine rapid barrier failure in chemical lung injury. JCI Insight 2019; 4: e124329. 
92 Uhl FE, Vierkotten S, Wagner DE, et al. Preclinical validation and imaging of Wnt-induced repair in human 3D lung tissue cultures. Eur Respir J 2015; 46: 1150-1166.

93 Akram KM, Yates LL, Mongey R, et al. Live imaging of alveologenesis in precision-cut lung slices reveals dynamic epithelial cell behaviour. Nat Commun 2019; 10: 1178.

94 Yang L, Feuchtinger A, Moeller W, et al. Three-dimensional quantitative co-mapping of pulmonary morphology and nanoparticle distribution with cellular resolution in non-dissected murine lungs. ACS Nano 2018; 13 : 1029-1041.

95 Ryan AL, Ikonomou L, Atarod S, et al. Stem cells, cell therapies, and bioengineering in lung biology and diseases 2017. An Official American Thoracic Society Workshop report. Am J Respir Cell Mol Biol 2019; 61: 429-439. 